\title{
Alpha-synuclein: from secretion to dysfunction and death
}

\author{
0 Marques $^{1}$ and TF Outeiro*,1,2,3
}

The aggregation, deposition, and dysfunction of alpha-synuclein (aSyn) are common events in neurodegenerative disorders known as synucleinopathies. These include Parkinson's disease, dementia with Lewy bodies, and multiple system atrophy. A growing body of knowledge on the biology of aSyn is emerging and enabling novel hypotheses to be tested. In particular, the hypothesis that aSyn is secreted from neurons, thus contributing to the spreading of pathology not only in the brain but also in other organs, is gaining momentum. Nevertheless, the precise mechanism(s) of secretion, as well as the consequences of extracellular aSyn species for neighboring cells are still unclear. Here, we review the current literature and integrate existing data in order to propose possible mechanisms of secretion, cell dysfunction, and death. Ultimately, the complete understanding of these processes might open novel avenues for the development of new therapeutic strategies.

Cell Death and Disease (2012) 3, e350; doi:10.1038/cddis.2012.94; published online 19 July 2012

Subject Category: Neuroscience

Facts

- Alpha-synuclein is associated with both familial and idiopathic cases of Parkinson's disease.

- The precise function of alpha-synuclein remains equivocal.

- Alpha-synuclein misfolds and forms protein aggregates in Parkinson's disease and in various in vitro and in vivo models of synucleinopathies.

- Alpha-synuclein has been detected in human and mouse CSF and in the media of cultured cells.

\section{Open Questions}

- Is alpha-synuclein normally secreted from cells or is it released from dying/damaged cells? How is it secreted?

- What are the effects of secreted forms of alpha-synuclein?

- What is the toxic genus of alpha-synuclein?

- Is the spreading of alpha-synuclein pathology a prion-like phenomenon?

\section{Alpha-synuclein and Synucleinopathies}

Alpha-synuclein (aSyn) is a 140 amino-acid protein that was originally identified in association with synaptic vesicles in the presynaptic nerve terminal ${ }^{1}$ and has been shown to interact with membranes both in vitro and in vivo. ${ }^{2-4}$ It is highly abundant in the brain and also present in other tissues, including red blood cells. ${ }^{5-8}$ aSyn is a member of a protein family of synucleins, together with beta $(\beta)$ - and gamma $(\gamma)$ synuclein. These proteins share a characteristic consensus sequence (KTKEGV) that is repeated about six times at the $\mathrm{N}$-terminal part of the protein. $\beta$-synuclein shares the closest homology $(90 \%$ homology in the $\mathrm{N}$-terminus and $33 \%$ homology in the C-terminus) with aSyn. ${ }^{5}$

Point mutations in the SNCA gene, encoding for aSyn, and multiplications of the SNCA locus have been identified in families with autosomal-dominant forms of Parkinson's disease (PD). ${ }^{9}$ Genome-wide association studies linked singlenucleotide polymorphisms in the SNCA gene with increased susceptibility to sporadic PD. ${ }^{10}$ Moreover, SNCA gene polymorphisms have also been associated with increased risk of multiple system atrophy (MSA). ${ }^{11}$

In PD, aSyn is found as a major component of Lewy bodies and Lewy neurites, the hallmark protein inclusions made up primarily of insoluble and fibrillar aSyn protein. ${ }^{12}$ aSyn also accumulates in dementia with Lewy bodies (DLB) and MSA. ${ }^{13}$ In MSA, aSyn is found predominantly within oligodendrocytes as cytoplasmic inclusions. ${ }^{14}$ These disorders share the accumulation of aSyn aggregates as a pathological feature and are collectively known as synucleinopathies. Additionally, aSyn was also identified as a component of amyloid from brain tissues of Alzheimer's disease (AD) patients. ${ }^{15}$

\footnotetext{
${ }^{1}$ Cell and Molecular Neuroscience Unit, Instituto de Medicina Molecular, Lisboa, Portugal; ${ }^{2}$ Instituto de Fisiologia, Faculdade de Medicina da Universidade de Lisboa, Lisboa, Portugal and ${ }^{3}$ Department of Neurodegeneration and Restorative Research, University Medizin Goettingen, Goettingen, Germany

${ }^{*}$ Corresponding author: TF Outeiro, Department of NeuroDegeneration and Restorative Research, University Medizin Gottingen, Waldweg 33, Gottingen 37073, Germany. Tel: +49 5513913544; Fax: +49 5513922693; E-mail: touteir@gwdg.de

Keywords: alpha-synuclein; Parkinson's disease; secretion; cell death; neuronal dysfunction

Abbreviations: aSyn, alpha-synuclein; PD, Parkinson's disease; DLB, dementia with Lewy bodies; SNP, single-nucleotide polymorphisms; MSA, multiple system atrophy; AD, Alzheimer's disease; $A \beta$, amyloid-beta peptide; PRA1, prenylated Rab acceptor protein 1; GDI, GDP dissociation inhibitors; CSP $\alpha$, cysteine-string protein alpha; LTP, long-term potentiation; CSF, cerebrospinal fluid; VPS, vacuolar protein sorting

Received 11.5.12; revised 18.6.12; accepted 20.6.12; Edited by G Melino
} 


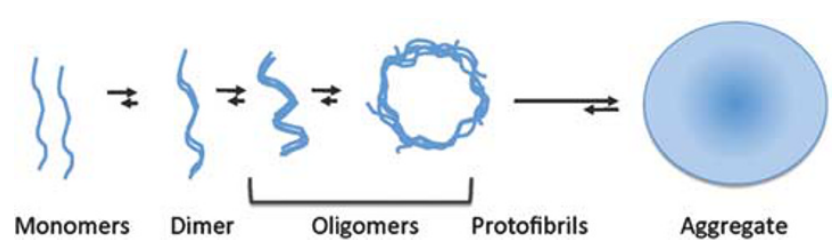

Figure 1 Schematic representation of the aSyn aggregation process. Monomeric forms of aSyn associate to form dimers and oligomers that grow into protofibrils and, finally, form mature fibrillar structures

The presence of a hydrophobic 12 amino-acid sequence in the central part of the protein is required for the oligomerization and fibrillization of aSyn. ${ }^{16}$ Deletion or disruption of this domain blocks the capacity of aSyn to form amyloid fibrils. The process of aSyn aggregation (Figure 1) has been studied in detail in an attempt to identify the toxic species responsible for neuronal dysfunction and death. However, it is still unclear what is/are the toxic forms of the protein. There is evidence showing that inhibition of aSyn aggregation process is associated with a decrease of aSyn toxicity. ${ }^{17,18}$ However, similarly to the case of amyloid-beta $(A \beta)$ plaques in $A D$, it was suggested that the fibrillar forms of aSyn might not represent the most toxic aSyn species. Instead, pre-fibrillar, soluble oligomeric species (comprising multiple aSyn molecules) are now suggested to be the main toxic aSyn species, with amyloid aggregates possibly serving as a reservoir for these oligomeric species (Figure 1). In vitro studies showed that the acceleration of oligomerisation, and not fibrilization, is the distinctive shared property of the A53T and A30P aSyn mutations linked to early-onset PD. ${ }^{19}$ The neurotoxic effects of aSyn oligomers were also studied in vivo, using animal models of synucleinopathies. In these studies, aSyn mutant variants that promote oligomer formation were designed and tested for toxicity in vivo. In one of these studies, overexpression of aSyn variants with impaired fibril formation ability caused increased toxicity in $C$. elegans and Drosophila models. Furthermore, the increasing inability of the mutants to form fibrils was directly correlated with toxicity and neurodegeneration. ${ }^{20}$ In another study, aSyn variants that were shown to promote oligomer formation caused most prominent dopaminergic cell death upon lentiviral injection into rat substantia nigra (SN). ${ }^{21}$ Together, these studies provide evidence for the importance of soluble oligomers as the prominent toxic species in synucleinopathies, although the precise size and type of the toxic oligomeric species remains to be determined.

Recently, it was hypothesized that PD, as well as other neurodegenerative disorders associated with protein misfolding and aggregation, might be a prion-like disease where pathological forms of aSyn spread throughout the brain. In particular, the spreading of aSyn pathology could contribute to the progression of neurodegeneration and clinical symptoms. ${ }^{22-24}$ Nevertheless, the molecular determinants underlying aSyn secretion, extracellular effects and transmission of pathology are still unclear.

\section{aSyn Function in Vesicle Trafficking}

Accumulating evidence from studies involving different model organisms, including yeast, worm, fly, and mammalian PD models, shows an association between aSyn and defects in vesicle trafficking.

aSyn was shown to induce aggregation of several yeast Rab GTPase proteins and this aggregation was more pronounced in yeast mutants that produce high levels of acidic phospholipids. ${ }^{25}$ Furthermore, aSyn overexpression in yeast causes cytoplasmic lipid droplet and vesicle accumulation. This effect is associated with an aSyn-induced block of ER-to-Golgi trafficking. ${ }^{26-28}$ Importantly, results from a genetic screen in yeast identified modifiers of ER-to-Golgi trafficking block as the most prominent suppressors of aSyn toxicity. ${ }^{27}$ The Rab GTPase Ypt1p (orthologue of the mammalian Rab1) was among the strongest suppressors, restoring normal ER-to-Golgi complex trafficking and aSyn localization to the plasma membrane. ${ }^{27}$ The protective effect of Rab1 against aSyn toxicity was further verified in rat primary midbrain neuronal cultures and in a Drosophila and a $C$. elegans model of PD, where Rab1 overexpression significantly reduced dopamine (DA) neuron cell death. ${ }^{27}$

An inhibitory effect of aSyn on ER-to-Golgi complex trafficking was verified also in mammalian kidney and neuroendocrine cells, with the A53T aSyn mutant causing stronger inhibition then the wild-type form. ${ }^{29}$ The aSyn-elicited trafficking defect can be rescued by the co-overexpression of Ykt6p, a vesicle-associated SNARE that promotes vesicle fusion, and has been amongst the suppressors of aSyn toxicity previously identified in yeast. 27,29

The mechanism of the aSyn-induced trafficking block was further addressed in a recent study showing that in neuronal cells, aSyn co-localizes and interacts with prenylated Rab acceptor protein 1 (PRA1). ${ }^{30}$ PRA1 is conserved from yeast to human and localizes predominantly in the Golgi and late endosomes. ${ }^{31-33}$ It was shown to interact with multiple prenylated Rab GTPases and regulate their recycling through inhibiting the removal of Rab from the membrane by GDP dissociation inhibitors. ${ }^{34}$ In addition to the previously described accumulation of cytoplasmatic vesicles upon aSyn overexpression, these vesicles localize in the periphery of the cytosol when aSyn is co-expressed together with PRA1. ${ }^{30}$ These results suggest a synergistic action of aSyn and PRA1 in interfering with vesicle trafficking and recycling.

In addition, aSyn was recently shown to induce vulnerability to perturbations in retrograde endosome-Golgi transport pathway in a yeast model. ${ }^{25}$

Together these studies suggest that aSyn causes defects in multiple vesicle trafficking steps.

\section{Effects of aSyn on Neuronal Function and Synaptic Transmission}

Although the physiological function of aSyn is not fully understood, there is evidence suggesting that aSyn has a role in neurotransmitter release $e^{35-38}$ and in the maintenance of the reserve pool of synaptic vesicles in primary hippocampal neurons.

Mice lacking the synaptic co-chaperone cysteine-string protein alpha (CSP $\alpha$ ) present progressive neurodegeneration and impairment in synaptic function. Interestingly, transgenic expression of aSyn rescued neurodegeneration and motor impairment resulting from $\operatorname{CSP} \alpha$ deficiency. Moreover, aSyn 
was able to reverse the SNARE-complex assembly impairment observed in the $\operatorname{CSP} \alpha$ knockout mice. ${ }^{36}$ These data indicate a physiological neuroprotective function of aSyn at the synapse.

Recently, a direct interaction between aSyn and SNARES was demonstrated. ${ }^{39}$ aSyn was found to bind the N-terminus of SNARE protein synaptobrevin-2 directly by its C-terminus. This study also brought additional evidence that aSyn enhances SNARE-complex assembly both in vitro and in vivo. ${ }^{39}$ Loss of synuclein function leads to an age-dependent impairment of SNARE-complex assembly and loss of neuronal function as shown in alpha-, $\beta$ - and $\gamma$-synuclein triple knockout mice. ${ }^{40}$

However, another study suggested an indirect inhibitory effect of aSyn on SNARE-complex assembly through sequestering of arachidonic acid. Arachidonic acid was shown to stimulate SNARE-complex formation and exocytosis and these effects were negatively regulated by aSyn both in vitro and in vivo. ${ }^{41}$

Together, these studies suggest a physiological function of aSyn at the synapse in modulating SNARE-complex assembly.

The role of aSyn in neurotransmitter release is mainly based on its regulation of synaptic vesicle recycling. aSyn knockout mice display altered DA release in response to paired stimuli, reduction in striatal DA and an attenuation of DA locomotor response to amphetamine. ${ }^{42}$ Another in vivo study showed that lack of aSyn leads to a permanent increase of the vesicle refilling rate in the DA readily releasable pool, maintaining stable DA release during stimulation in contrast to decline of DA release observed in normal conditions. ${ }^{43}$ Together, these findings suggest that aSyn is an activitydependent, negative regulator of DA neurotransmission. Moreover, aSyn has also been shown to regulate synaptic glutamate release. Electrophysiology studies in brains from aSyn knockout mice suggest that lack of aSyn impairs mobilization of glutamate from the reserve pool. ${ }^{44}$

Studies in animal models of PD pathology implicate aSyn in functional synaptic deficits. In a transgenic mouse model overexpressing A30P mutant aSyn, a reduction of locomotor activity accompanied by a reduced size of the DA storage pool was observed. ${ }^{45}$ In the striatum of a transgenic mouse model expressing truncated human aSyn, synaptic accumulation of aSyn was accompanied by an age-dependent redistribution of the synaptic SNARE proteins as well as by reduction in DA release. ${ }^{46}$ This redistribution of SNAREs is also observed in PD brain samples. In cultured neurons from another PD mouse model, aSyn overexpression leads to loss of several presynaptic proteins and neurotransmitter release deficits. ${ }^{47}$ A modest overexpression of aSyn that does not lead to overt toxicity causes significant inhibition of neurotransmitter release through inhibition of vesicle reclustering after endocytosis. ${ }^{48}$ These results indicate that increased levels of aSyn lead to a defect in synaptic vesicle recycling that precedes detectable neuropathology.

Imaging studies in patients also point to synaptic dysfunction and neurotransmitter deficiencies in synucleinopathies including PD and DLB. ${ }^{49}$ Recently, small aSyn detergentinsoluble aggregates with presynaptic localization were detected abundantly in patients with DLB, together with significant synaptic pathology with almost complete loss of dendritic spines at the postsynaptic area. ${ }^{50,51}$ It has been suggested that it is the presynaptic aSyn aggregation-linked synaptic dysfunction, rather than cell death, that leads to neurodegeneration in DLB and PD. ${ }^{51}$

Recently, the impact of different aSyn species (monomers, oligomers, and fibrils) on synaptic transmission was explored. Interestingly, oligomeric forms of aSyn were shown to increase synaptic transmission and to impair long-term potentiation, $^{52}$ a paradigm that mimics the process of memory formation. The effects of aSyn were mediated by NMDA receptor activation, in similarity to what is also known for $\mathrm{A} \beta$ oligomers (Diogenes and Outeiro, personal communication).

Altogether, the effects of aSyn on neuronal function are emerging and will provide important insight into the molecular basis of PD and other synucleinopathies.

\section{Secretion of aSyn and Disease Propagation}

The absence of a secretory signal peptide sequence in aSyn suggested it might be purely an intracellular protein and its pathological function was, therefore, studied in a cell autonomous context. However, this view was challenged by the presence of aSyn in biological fluids such as cerebrospinal fluid (CSF) and blood plasma of both PD and normal subjects. ${ }^{53-55}$ Recently, aSyn release mechanisms as well as possible functions of extracellular aSyn have been intensively studied.

aSyn was shown to be secreted into the culture medium in various cell lines overexpressing aSyn. ${ }^{53,56,57}$ Moreover, secretion of endogenous aSyn from rat embryonic cortical neurons was also demonstrated. ${ }^{57}$ Both monomeric and aggregated forms of aSyn were found to be secreted (Figure 2). ${ }^{57,58}$

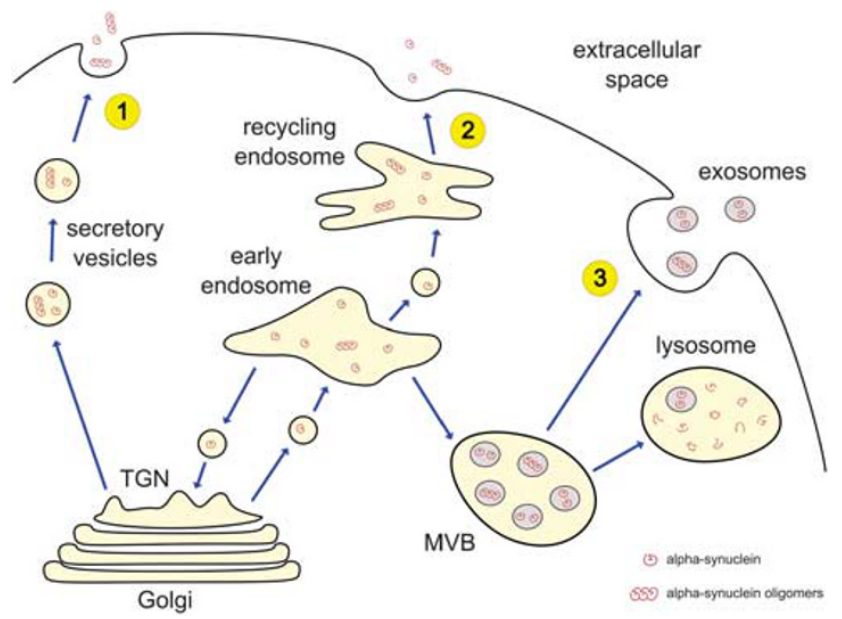

Figure 2 Proposed mechanisms of aSyn secretion. aSyn can be secreted both in its monomeric or aggregated forms by non-classical exocytic or endocytic pathways. aSyn can be directly integrated into secretory vesicles and subsequently released by exocytosis (1). Alternatively, aSyn can be translocated to early endosomes. From early endosomes, aSyn protein can either be released to the extracellular space through the recycling endosome (2) or incorporated to intraluminal vesicles of multivesicular bodies (MBV). MVB cargo including aSyn can be directed to degradation by fusion with lysosomes or to secretion by fusion with the plasma membrane and release of exosomal vesicles (3). TGN = trans-Golgi network 
The mechanisms of aSyn secretion are not fully understood, but there is evidence showing that aSyn can be secreted by non-classical, ER/Golgi-independent protein export pathway (Figure 2). ${ }^{57}$ Upon overexpression in SH-SY5Y cells, aSyn localizes in large dense core vesicles. Interestingly, it was shown that this intravesicular fraction of aSyn is more prone to aggregation compared with cytosolic aSyn protein. ${ }^{57}$ In another study, DA was shown to induce aSyn aggregation in the vesicle fraction and to increase aSyn secretion levels. ${ }^{59}$ It has also been suggested that the release of aSyn is upregulated under cellular stress conditions that cause accumulation of misfolded/damaged proteins. ${ }^{60}$

Additionally, a fraction of aSyn was shown to be secreted in association with exosomes by a calcium-dependent mechanism (Figure 2) ${ }^{61}$ and exosomal aSyn release was found to be increased upon lysosomal dysfunction. ${ }^{62}$ However, a very recent study reports no enrichment of aSyn in exosomal pellet of neuronal cells overexpressing aSyn as well as an absence of aSyn-positive signal in exosomal fraction derived from CSF of PD patients and control subjects. ${ }^{63}$ This study further shows that in neuronal cells aSyn is present in endosomal compartments (Figure 2), and that aSyn secretion and lysosomal targeting is regulated by vacuolar protein sorting 4. Moreover, it was suggested that the recycling pathway regulated by Rab11 has functional relevance in aSyn secretion (Figure 2). ${ }^{63}$

In recent years, extracellular aSyn has received a lot of scientific attention due to its potential role in disease initiation and progression. Considering the progressive nature of neurodegenerative disorders as well as the defined, stepwise spreading of Lewy body pathology in $\mathrm{PD},{ }^{22}$ the idea of extracellular aSyn as a pathogenic 'prion-like' agent is extremely appealing. This scenario is strengthened by observations describing the presence of aSyn-positive Lewy-body-like inclusions in long-term mesencephalic transplants in PD patients. ${ }^{23,64}$ These findings suggest host-tograft propagation of aSyn-associated pathology. Subsequent in vitro experiments showed inter-neuronal transmission of aSyn (Figure 3). ${ }^{58,65}$ Moreover, the host-to-graft transmission of aSyn has also been observed in vivo in aSyn transgenic mouse models. ${ }^{24,65}$ This transmission process seems to rely on endocytic uptake of aSyn by the recipient neurons. ${ }^{24,66}$

All of these findings indicate that aSyn can be both secreted and transmitted between neuronal cells. Moreover, there is evidence suggesting that aSyn secretion is stimulated by cellular stress conditions including lysosomal dysfunction.

\section{aSyn-induced Neuroinflammation and Cell Death}

Although many studies showed secretion and cell-to-cell transmission of aSyn, this phenomenon might not, per se, be sufficient to explain the spread of aSyn Lewy-body-like pathology and progressive neurodegeneration observed in PD. There is additional evidence, however, that aSyn can act as a nucleation/seeding agent for aggregation and can affect the viability of the recipient neurons (Figure 3).

Exogenous aSyn fibrils were shown to seed the formation of Lewy-body-like intracellular inclusions in cultured cells. ${ }^{67}$ Furthermore, there is evidence of inclusion formation via aSyn cell-to-cell transmission both in cell co-cultures and

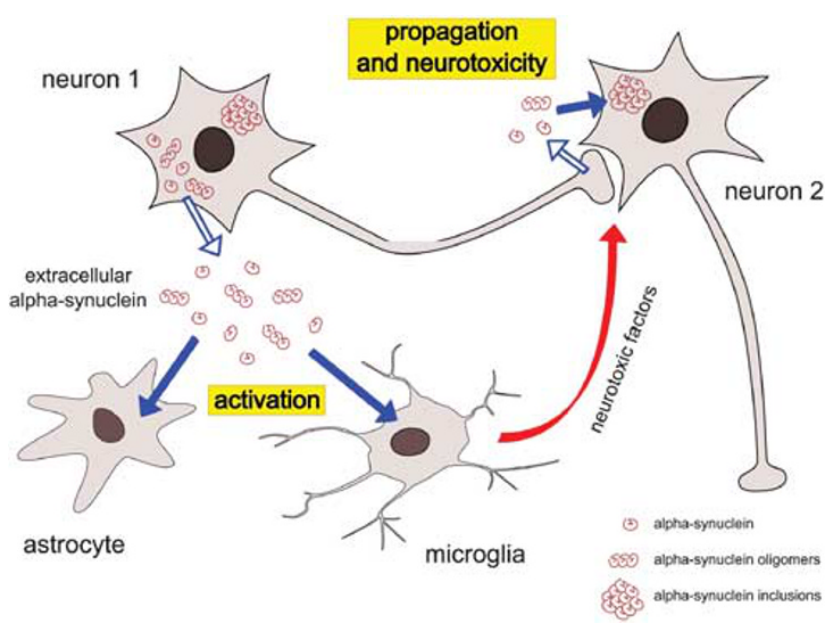

Figure 3 The role of extracellular aSyn in neuroinflammation, neurotoxicity, and spread of pathology. aSyn can be actively secreted or released by dying neurons to the extracellular space. Extracellular aSyn can then activate surrounding astrocytes and microglia, eliciting glial pro-inflammatory activity. Upon activation microglia produce pro-inflammatory cytokines, nitric oxide, and reactive oxygen species, which may be toxic to neurons. aSyn can be directly transferred between neurons, leading to propagation of aSyn aggregation process and compromising the viability of the recipient neuron

in vivo in grafted cells (Figure 3). ${ }^{24,65}$ Importantly, in addition to seeding aSyn aggregation, neuron-derived extracellular aSyn triggered apoptotic process in recipient neurons. ${ }^{65}$

The neurotoxic effect of extracellular aSyn oligomeric species was addressed in several studies. Recombinant aSyn oligomers can be taken up by neurons in culture and trigger cell death. ${ }^{68,69}$ Furthermore, aSyn oligomers secreted from neuronal cells were also shown to induce toxicity in recipient neurons, ${ }^{58}$ an effect that was inhibited by Hsp70. Moreover, treatment of the conditioned media containing neuron-derived aSyn with oligomer-interfering compounds rescued the recipient neuronal cells from toxicity. ${ }^{61}$ Together, these studies indicate an important role of extracellular aSyn in the progression of PD-linked pathological processes, propagation of inclusion bodies and neurodegeneration.

The importance of extracellular aSyn in PD pathology is further supported by the role of aSyn in triggering neuroinflammatory glial responses. Several studies indicate that an inflammatory process in the $\mathrm{SN}$, characterized by the presence of activated microglia and the secretion of proinflammatory and neurotoxic factors, may contribute to either initiation and/or aggravation of neuronal degeneration. ${ }^{70-72}$ Studies of biological fluids from PD patients further support a role for neuroinflammatory processes in PD, as increased levels of pro-inflammatory cytokines such as interleukin-1 $\beta$ (IL1- $\beta$ ), interleukin-2 (IL-2), interleukin-6 (IL-6) or tumor necrosis factor-alpha (TNF- $\alpha$ ) were found in CSF or serum of PD patients. ${ }^{73-75}$

Various other studies focused on the investigation of the extracellular effects of aSyn on glial cell activation (Figure 3). ${ }^{76-80}$ Upon aSyn exposure in vitro, microglia cells produce increased levels of NO, TNF- $\alpha$ and IL1- $\beta^{77,78}$. The $\mathrm{A} 30 \mathrm{P}$ and E46K mutants of aSyn were shown to elicit stronger pro-inflammatory and immunoregulatory responses when 
compared with wild-type aSyn. ${ }^{81}$ In addition, the induction of microglia classical activation by extracellular aSyn also includes modulation of toll-like receptor expression. ${ }^{77}$ Extracellular aggregated aSyn added to neuron-glia culture lead to microglia activation and enhanced dopaminergic neurodegeneration (Figure 3). ${ }^{76}$ In this study, microglial enhancement of aSyn-mediated neurotoxicity was shown to be dependent on phagocytosis of aSyn and activation of NADPH oxidase with production of reactive oxygen species. ${ }^{76}$ Recently, direct activation of the inflammatory response in a microglial cell line by neuron-derived extracellular aSyn was described. ${ }^{82}$

A direct effect of extracellular aSyn on microglia activation in vivo was described in mice injected with human wild-type aSyn protein into the $\mathrm{SN}$. This treatment induced expression of pro-inflammatory cytokines, the expression of endothelial markers of inflammation and microglial activation. ${ }^{78}$ Moreover, it was demonstrated that the presence of extracellular aSyn made microglia more susceptible to systemic proinflammatory challenge. ${ }^{78}$

In addition to the effects on microglia, aSyn has also been shown to induce astrocytic responses (Figure 3). Transfer of aSyn from neurons to astrocytes was demonstrated in culture as well as in vivo in brains of transgenic mice expressing human aSyn. ${ }^{79}$ The transmission of neuronal aSyn led to intra-astrocytic aSyn accumulation and triggered astrocytic inflammatory response.

Together these different studies provide evidence for the role of extracellular aSyn in modulating glial function and neuroinflammation, further supporting the importance of the study of the mechanisms by which aSyn reaches extracellular compartments.

\section{Therapeutic Intervention and Future Perspectives}

The process of aSyn aggregation is thought to be associated with both a gain of toxic function and to haploinsufficiency, by sequestering the wild-type protein and therefore reducing the amount of functional aSyn available in the cell. Although accumulating evidence suggests oligomeric forms of aSyn might constitute the toxic genus, it is still currently unclear what is the precise form of the toxic aSyn species. Thus, one possible intervention strategy might be to interfere with aSyn aggregation in order to reduce the levels of the toxic species.

Recently, aSyn was suggested to exist, physiologically, as a folded tetramer. ${ }^{83}$ Moreover, it was shown that the tetrameric form of aSyn resists aggregation. Interestingly, the stabilization of the native structure of proteins, preventing their initial misfolding, is an appealing intervention strategy. For oligomeric proteins, such as transthyretin, for example, compounds capable of stabilizing the functional tetrameric structure hold great potential and are in the process of being developed. ${ }^{84,85}$ In light of the recent findings for aSyn, and despite the current controversy in the field, it is tempting to speculate that a similar strategy might be envisioned for PD and other synucleinopathies.

Another line of possible intervention is linked with the emerging role of extracellular aSyn in disease progression. Interfering with either the process of aSyn secretion or with the neuronal uptake of extracellular aSyn might prove beneficial for stopping or slowing down the propagation of
aSyn pathology in PD and other synucleinopathies. In addition, removal of aSyn from the extracellular space might decrease detrimental inflammatory processes. One promising approach is immunotherapy, as immunization with human aSyn reduces the accumulation of aggregated aSyn and reduces neurodegeneration in transgenic mice overexpressing aSyn. ${ }^{86}$ Thus, immunotherapy with antibodies promoting the removal of extracellular aSyn species might delay the progression of the disease.

Altogether, the recent progress in our understanding of the molecular mechanisms underlying aSyn secretion and cytotoxicity might pave the way for the development of novel strategies for therapeutic intervention in PD and other synucleinopathies.

\section{Conflict of Interest}

The authors declare no conflict of interest.

Acknowledgements. TFO is supported by Fundação para a Ciência e Tecnologia (PTDC/SAU-NEU/105215/2008) and an EMBO Installation Grant. OM is supported by a fellowship from Fundação para a Ciência e Tecnologia (SFRH/BD/44446/2008).

1. Maroteaux L, Campanelli JT, Scheller RH. Synuclein: a neuron-specific protein localized to the nucleus and presynaptic nerve terminal. J Neurosci 1988; 8: 2804-2815.

2. Davidson WS, Jonas A, Clayton DF, George JM. Stabilization of alpha-synuclein secondary structure upon binding to synthetic membranes. J Biol Chem 1998; 273: 9443-9449.

3. Jo E, McLaurin J, Yip CM St, George-Hyslop P, Fraser PE. alpha-Synuclein membrane interactions and lipid specificity. J Biol Chem 2000; 275: 34328-34334.

4. Fortin DL, Troyer MD, Nakamura K, Kubo S, Anthony MD, Edwards RH. Lipid rafts mediate the synaptic localization of alpha-synuclein. J Neurosci 2004; 24: 6715-6723.

5. Jakes R, Spillantini MG, Goedert M. Identification of two distinct synucleins from human brain. FEBS Lett 1994; 345: 27-32.

6. Iwai A, Masliah E, Yoshimoto M, Ge N, Flanagan L, de Silva HA et al. The precursor protein of non-A beta component of Alzheimer's disease amyloid is a presynaptic protein of the central nervous system. Neuron 1995; 14: 467-475.

7. Nakai M, Fujita M, Waragai M, Sugama S, Wei J, Akatsu $\mathrm{H}$ et al. Expression of alphasynuclein, a presynaptic protein implicated in Parkinson's disease, in erythropoietic lineage. Biochem Biophys Res Commun 2007; 358: 104-110.

8. Barbour R, Kling K, Anderson JP, Banducci K, Cole T, Diep L et al. Red blood cells are the major source of alpha-synuclein in blood. Neuro-degener Dis 2008; 5: 55-59.

9. Hardy J, Lewis P, Revesz T, Lees A, Paisan-Ruiz C. The genetics of Parkinson's syndromes: a critical review. Curr Opin Genetics Dev 2009; 19: 254-265.

10. Nalls MA, Plagnol V, Hernandez DG, Sharma M, Sheerin UM, Saad M et al. Imputation of sequence variants for identification of genetic risks for Parkinson's disease: a metaanalysis of genome-wide association studies. Lancet 2011; 377: 641-649.

11. Scholz SW, Houlden H, Schulte C, Sharma M, Li A, Berg D et al. SNCA variants are associated with increased risk for multiple system atrophy. Ann Neurol 2009; 65: 610-614.

12. Spillantini MG, Crowther RA, Jakes R, Hasegawa M, Goedert M. alpha-Synuclein in filamentous inclusions of Lewy bodies from Parkinson's disease and dementia with lewy bodies. Proc Natl Acad Sci USA 1998; 95: 6469-6473.

13. Spillantini MG, Crowther RA, Jakes R, Cairns NJ, Lantos PL, Goedert M. Filamentous alpha-synuclein inclusions link multiple system atrophy with Parkinson's disease and dementia with Lewy bodies. Neurosci Lett 1998; 251: 205-208.

14. Wenning GK, Geser F. Multiple system atrophy. Revue Neurologique 2003; 159(5 Pt 2): 3S31-38.

15. Ueda K, Fukushima H, Masliah E, Xia Y, Iwai A, Yoshimoto M et al. Molecular cloning of cDNA encoding an unrecognized component of amyloid in Alzheimer disease. Proc Natl Acad Sci USA 1993; 90: 11282-11286.

16. Giasson BI, Murray IV, Trojanowski JQ, Lee VM. A hydrophobic stretch of 12 amino acid residues in the middle of alpha-synuclein is essential for filament assembly. J Biol Chem 2001; 276: 2380-2386.

17. Hashimoto M, Rockenstein E, Mante M, Mallory M, Masliah E. beta-Synuclein inhibits alpha-synuclein aggregation: a possible role as an anti-Parkinsonian factor. Neuron 2001; 32: 213-223.

18. Periquet M, Fulga T, Myllykangas L, Schlossmacher MG, Feany MB. Aggregated alphasynuclein mediates dopaminergic neurotoxicity in vivo. J Neurosci 2007; 27: 3338-3346. 
19. Conway KA, Lee SJ, Rochet JC, Ding TT, Williamson RE, Lansbury PT Jr.. Acceleration of oligomerization, not fibrillization, is a shared property of both alpha-synuclein mutations linked to early-onset Parkinson's disease: implications for pathogenesis and therapy. Proc Natl Acad Sci USA 2000; 97: 571-576.

20. Karpinar DP, Balija MB, Kugler S, Opazo F, Rezaei-Ghaleh N, Wender N et al. Pre-fibrillar alpha-synuclein variants with impaired beta-structure increase neurotoxicity in Parkinson's disease models. EMBO J 2009; 28: 3256-3268.

21. Winner B, Jappelli R, Maji SK, Desplats PA, Boyer L, Aigner S et al. In vivo demonstration that alpha-synuclein oligomers are toxic. Proc Natl Acad Sci USA 2011; 108: 4194-4199.

22. Braak H, Del Tredici K, Rub U, de Vos RA, Jansen Steur EN, Braak E. Staging of brain pathology related to sporadic Parkinson's disease. Neurobiol Aging 2003; 24: 197-211.

23. Li JY, Englund E, Holton JL, Soulet D, Hagell P, Lees AJ et al. Lewy bodies in grafted neurons in subjects with Parkinson's disease suggest host-to-graft disease propagation. Nat Med 2008; 14: 501-503.

24. Hansen C, Angot E, Bergstrom AL, Steiner JA, Pieri L, Paul G et al. alpha-Synuclein propagates from mouse brain to grafted dopaminergic neurons and seeds aggregation in cultured human cells. J Clin Invest 2011; 121: 715-725.

25. Soper JH, Kehm V, Burd CG, Bankaitis VA, Lee VM. Aggregation of alpha-synuclein in S. cerevisiae is associated with defects in endosomal trafficking and phospholipid biosynthesis. J Mol Neurosci, 2011; 43: 391-405.

26. Outeiro TF, Lindquist S. Yeast cells provide insight into alpha-synuclein biology and pathobiology. Science 2003; 302: 1772-1775.

27. Cooper AA, Gitler AD, Cashikar A, Haynes CM, Hill KJ, Bhullar B et al. Alpha-synuclein blocks ER-Golgi traffic and Rab1 rescues neuron loss in Parkinson's models. Science 2006; 313: 324-328.

28. Gitler AD, Bevis BJ, Shorter J, Strathearn KE, Hamamichi S, Su LJ et al. The Parkinson's disease protein alpha-synuclein disrupts cellular Rab homeostasis. Proc Natl Acad Sci USA 2008; 105: 145-150.

29. Thayanidhi N, Helm JR, Nycz DC, Bentley M, Liang Y, Hay JC. Alpha-synuclein delays endoplasmic reticulum (ER)-to-Golgi transport in mammalian cells by antagonizing ER/ Golgi SNAREs. Mol Biol Cell 2010; 21: 1850-1863.

30. Lee HJ, Kang SJ, Lee K, Im H. Human alpha-synuclein modulates vesicle trafficking through its interaction with prenylated Rab acceptor protein 1. Biochem Biophys Res Commun 2011; 412: 526-531.

31. Bucci $C$, Chiariello M, Lattero D, Maiorano M, Bruni $C B$. Interaction cloning and characterization of the cDNA encoding the human prenylated rab acceptor (PRA1) Biochem Biophys Res Commun 1999; 258: 657-662.

32. Abdul-Ghani M, Gougeon PY, Prosser DC, Da-Silva LF, Ngsee JK. PRA isoforms are targeted to distinct membrane compartments. J Biol Chem 2001; 276: 6225-6233.

33. Sivars U, Aivazian D, Pfeffer SR. Yip3 catalyses the dissociation of endosomal Rab-GDI complexes. Nature 2003; 425: 856-859.

34. Hutt DM, Da-Silva LF, Chang LH, Prosser DC, Ngsee JK. PRA1 inhibits the extraction of membrane-bound rab GTPase by GDI1. J Biol Chem 2000; 275: 18511-18519.

35. Murphy DD, Rueter SM, Trojanowski JQ, Lee VM. Synucleins are developmentally expressed, and alpha-synuclein regulates the size of the presynaptic vesicular pool in primary hippocampal neurons. J Neurosci 2000; 20: 3214-3220.

36. Chandra S, Gallardo G, Fernandez-Chacon R, Schluter OM, Sudhof TC. Alpha-synuclein cooperates with CSPalpha in preventing neurodegeneration. Cell 2005; 123: 383-396.

37. Fortin DL, Nemani VM, Voglmaier SM, Anthony MD, Ryan TA, Edwards RH. Neural activity controls the synaptic accumulation of alpha-synuclein. J Neurosci 2005; 25 10913-10921.

38. Liu S, Ninan I, Antonova I, Battaglia F, Trinchese F, Narasanna A et al. alpha-Synuclein produces a long-lasting increase in neurotransmitter release. EMBO J 2004; 23 4506-4516.

39. Burre J, Sharma M, Tsetsenis T, Buchman V, Etherton MR, Sudhof TC. Alpha-synuclein promotes SNARE-complex assembly in vivo and in vitro. Science 2010; 329: 1663-1667.

40. Greten-Harrison B, Polydoro M, Morimoto-Tomita M, Diao L, Williams AM, Nie EH et al. alphabetagamma-Synuclein triple knockout mice reveal age-dependent neuronal dysfunction. Proc Natl Acad Sci USA 2010; 107: 19573-19578.

41. Darios F, Ruiperez V, Lopez I, Villanueva J, Gutierrez LM, Davletov B. Alpha-synuclein sequesters arachidonic acid to modulate SNARE-mediated exocytosis. EMBO Rep 2010; 11: $528-533$.

42. Abeliovich A, Schmitz Y, Farinas I, Choi-Lundberg D, Ho WH, Castillo PE et al. Mice lacking alpha-synuclein display functional deficits in the nigrostriatal dopamine system. Neuron 2000; 25: 239-252.

43. Yavich L, Tanila H, Vepsalainen S, Jakala $P$. Role of alpha-synuclein in presynaptic dopamine recruitment. J Neurosci 2004; 24: 11165-11170.

44. Gureviciene I, Gurevicius K, Tanila H. Role of alpha-synuclein in synaptic glutamate release. Neurobiol Dis 2007; 28: 83-89.

45. Yavich L, Oksman M, Tanila H, Kerokoski $\mathrm{P}$, Hiltunen $\mathrm{M}$, van Groen $\mathrm{T}$ et al. Locomotor activity and evoked dopamine release are reduced in mice overexpressing A30P-mutated human alpha-synuclein. Neurobiol Dis 2005; 20: 303-313.

46. Garcia-Reitbock $P$, Anichtchik O, Bellucci A, lovino M, Ballini C, Fineberg E et al. SNARE protein redistribution and synaptic failure in a transgenic mouse model of Parkinson's disease. Brain 2010; 133(Pt 7): 2032-2044.
47. Scott DA, Tabarean I, Tang Y, Cartier A, Masliah E, Roy S. A pathologic cascade leading to synaptic dysfunction in alpha-synuclein-induced neurodegeneration. J Neurosci 2010; 30: 8083-8095.

48. Nemani VM, Lu W, Berge V, Nakamura K, Onoa B, Lee MK et al. Increased expression of alpha-synuclein reduces neurotransmitter release by inhibiting synaptic vesicle reclustering after endocytosis. Neuron 2010; 65: 66-79.

49. Nikolaus S, Antke C, Muller HW. In vivo imaging of synaptic function in the central nervous system: I. Movement disorders and dementia. Behav Brain Res 2009; 204: 1-31.

50. Kramer ML, Behrens C, Schulz-Schaeffer WJ. Selective detection, quantification, and subcellular location of alpha-synuclein aggregates with a protein aggregate filtration assay. BioTechniques 2008; 44: 403-411.

51. Schulz-Schaeffer WJ. The synaptic pathology of alpha-synuclein aggregation in dementia with Lewy bodies, Parkinson's disease and Parkinson's disease dementia. Acta Neuropathologica 2010; 120: 131-143.

52. Martin ZS, Neugebauer V, Dineley KT, Kayed R, Zhang W, Reese LC et al. alphaSynuclein oligomers oppose long-term potentiation and impair memory through a calcineurin-dependent mechanism: relevance to human synucleopathic diseases. J Neurochem 2012; 120: 440-452.

53. El-Agnaf OM, Salem SA, Paleologou KE, Cooper LJ, Fullwood NJ, Gibson MJ et al. Alphasynuclein implicated in Parkinson's disease is present in extracellular biological fluids, including human plasma. FASEB J 2003; 17: 1945-1947.

54. Tokuda T, Salem SA, Allsop D, Mizuno T, Nakagawa M, Qureshi MM et al. Decreased alpha-synuclein in cerebrospinal fluid of aged individuals and subjects with Parkinson's disease. Biochem Biophys Res Commun 2006; 349: 162-166.

55. Lee PH, Lee G, Park HJ, Bang OY, Joo IS, Huh K. The plasma alpha-synuclein levels in patients with Parkinson's disease and multiple system atrophy. J Neural Transm 2006; 113: 1435-1439.

56. Sung JY, Park SM, Lee CH, Um JW, Lee HJ, Kim J et al. Proteolytic cleavage of extracellular secreted \{alpha\}-synuclein via matrix metalloproteinases. J Biol Chem 2005; 280: 25216-25224.

57. Lee HJ, Patel S, Lee SJ. Intravesicular localization and exocytosis of alpha-synuclein and its aggregates. J Neurosci 2005; 25: 6016-6024.

58. Danzer KM, Ruf WP, Putcha P, Joyner D, Hashimoto T, Glabe $C$ et al. Heat-shock protein 70 modulates toxic extracellular alpha-synuclein oligomers and rescues trans-synaptic toxicity. FASEB J 2011; 25: 326-336.

59. Lee HJ, Baek SM, Ho DH, Suk JE, Cho ED, Lee SJ. Dopamine promotes formation and secretion of non-fibrillar alpha-synuclein oligomers. Exp Mol Med 2011; 43: 216-222.

60. Jang A, Lee HJ, Suk JE, Jung JW, Kim KP, Lee SJ. Non-classical exocytosis of alphasynuclein is sensitive to folding states and promoted under stress conditions. J Neurochem 2010; 113: 1263-1274.

61. Emmanouilidou E, Melachroinou K, Roumeliotis T, Garbis SD, Ntzouni M, Margaritis LH et al. Cell-produced alpha-synuclein is secreted in a calcium-dependent manner by exosomes and impacts neuronal survival. J Neurosci 2010; 30: 6838-6851.

62. Alvarez-Erviti L, Seow Y, Schapira AH, Gardiner C, Sargent IL, Wood MJ et al. Lysosomal dysfunction increases exosome-mediated alpha-synuclein release and transmission. Neurobiol Dis 2011; 42: 360-367.

63. Hasegawa T, Konno M, Baba T, Sugeno N, Kikuchi A, Kobayashi M et al. The AAAATPase VPS4 regulates extracellular secretion and lysosomal targeting of alpha-synuclein PloS One 2011; 6: e29460.

64. Kordower JH, Chu Y, Hauser RA, Freeman TB, Olanow CW. Lewy body-like pathology in long-term embryonic nigral transplants in Parkinson's disease. Nat Med 2008; 14: 504-506.

65. Desplats P, Lee HJ, Bae EJ, Patrick C, Rockenstein E, Crews L et al. Inclusion formation and neuronal cell death through neuron-to-neuron transmission of alpha-synuclein. Proc Natl Acad Sci USA 2009; 106: 13010-13015.

66. Lee HJ, Suk JE, Bae EJ, Lee JH, Paik SR, Lee SJ. Assembly-dependent endocytosis and clearance of extracellular alpha-synuclein. Int J Biochem Cell Biol 2008; 40: 1835-1849.

67. Luk KC, Song C, O'Brien P, Stieber A, Branch JR, Brunden KR et al. Exogenous alphasynuclein fibrils seed the formation of Lewy body-like intracellular inclusions in cultured cells. Proc Natl Acad Sci USA 2009; 106: 20051-20056.

68. Danzer KM, Haasen D, Karow AR, Moussaud S, Habeck M, Giese A et al. Different species of alpha-synuclein oligomers induce calcium influx and seeding. $J$ Neurosci 2007; 27: 9220-9232.

69. Danzer KM, Krebs SK, Wolff M, Birk G, Hengerer B. Seeding induced by alpha-synuclein oligomers provides evidence for spreading of alpha-synuclein pathology. $J$ Neurochem 2009; 111: 192-203.

70. McGeer PL, Itagaki S, Boyes BE, McGeer EG. Reactive microglia are positive for HLA-DR in the substantia nigra of Parkinson's and Alzheimer's disease brains. Neurology 1988; 38: 1285-1291.

71. Croisier E, Moran LB, Dexter DT, Pearce RK, Graeber MB. Microglial inflammation in the Parkinsonian substantia nigra: relationship to alpha-synuclein deposition. J Neuroinflammation 2005; 2: 14.

72. Hirsch EC, Hunot S, Hartmann A. Neuroinflammatory processes in Parkinson's disease. Parkinsonism Relat Disord 2005; 11(Suppl 1): S9-S15.

73. Blum-Degen D, Muller T, Kuhn W, Gerlach M, Przuntek H, Riederer P. Interleukin-1 beta and interleukin- 6 are elevated in the cerebrospinal fluid of Alzheimer's and de novo Parkinson's disease patients. Neurosci Lett 1995; 202: 17-20. 
74. Mogi M, Harada M, Riederer P, Narabayashi H, Fujita K, Nagatsu T. Tumor necrosis factoralpha (TNF-alpha) increases both in the brain and in the cerebrospinal fluid from Parkinsonian patients. Neurosci Lett 1994; 165: 208-210.

75. Stypula G, Kunert-Radek J, Stepien H, Zylinska K, Pawlikowski M. Evaluation of interleukins, ACTH, cortisol and prolactin concentrations in the blood of patients with Parkinson's disease. Neuroimmunomodulation 1996; 3: 131-134.

76. Zhang W, Wang T, Pei Z, Miller DS, Wu X, Block ML et al. Aggregated alpha-synuclein activates microglia: a process leading to disease progression in Parkinson's disease. FASEB J 2005; 19: 533-542.

77. Beraud D, Twomey M, Bloom B, Mittereder A, Ton V, Neitzke K et al. alpha-Synuclein Alters Toll-Like Receptor Expression. Front Neurosci 2011; 5: 80.

78. Couch Y, Alvarez-Erviti L, Sibson NR, Wood MJ, Anthony DC. The acute inflammatory response to intranigral alpha-synuclein differs significantly from intranigral lipopolysaccharide and is exacerbated by peripheral inflammation. J Inflamm 2011; 8: 166

79. Lee HJ, Suk JE, Patrick C, Bae EJ, Cho JH, Rho S et al. Direct transfer of alpha-synuclein from neuron to astroglia causes inflammatory responses in synucleinopathies. J Biol Chem 2010; 285: 9262-9272.

80. Su X, Federoff HJ, Maguire-Zeiss KA. Mutant alpha-synuclein overexpression mediates early proinflammatory activity. Neurotox Res 2009; 16: 238-254.

81. Roodveldt C, Labrador-Garrido A, Gonzalez-Rey E, Fernandez-Montesinos R, Caro M Lachaud CC et al. Glial innate immunity generated by non-aggregated alpha-synuclein in mouse: differences between wild-type and Parkinson's disease-linked mutants. PloS One 2010; 5: e13481.
82. Alvarez-Erviti L, Couch Y, Richardson J, Cooper JM, Wood MJ. Alpha-synuclein release by neurons activates the inflammatory response in a microglial cell line. Neurosci Res 2011; 69: 337-342.

83. Bartels T, Choi JG, Selkoe DJ. alpha-Synuclein occurs physiologically as a helically folded tetramer that resists aggregation. Nature 2011; 477: 107-110.

84. Wiseman RL, Green NS, Kelly JW. Kinetic stabilization of an oligomeric protein unde physiological conditions demonstrated by a lack of subunit exchange: implications for transthyretin amyloidosis. Biochemistry 2005; 44: 9265-9274.

85. Petrassi HM, Johnson SM, Purkey $\mathrm{HE}$, Chiang KP, Walkup $\mathrm{T}$, Jiang $\mathrm{X}$ et al. Potent and selective structure-based dibenzofuran inhibitors of transthyretin amyloidogenesis: kinetic stabilization of the native state. J Am Chem Soc 2005; 127: 6662-6671.

86. Masliah E, Rockenstein E, Adame A, Alford M, Crews L, Hashimoto M et al. Effects of alpha-synuclein immunization in a mouse model of Parkinson's disease. Neuron 2005; 46: 857-868.

Cell Death and Disease is an open-access journal published by Nature Publishing Group. This work is licensed under the Creative Commons Attribution-NonCommercial-No Derivative Works 3.0 Unported License. To view a copy of this license, visit http://creativecommons.org/licenses/by-nc-nd/3.0/ 